\title{
Conducting power flow analysis and simulations for a delivery electric system with important consumers
}

\author{
Mariana Dumitrescu ${ }^{1 *}$ \\ ${ }^{1}$ University Dunarea de Jos, Automation and Electric Engineering Department, Stiintei 02, Galati, \\ Romania
}

\begin{abstract}
The goals of the paper are to conduct the simulation and to analyze the power flow in a real complex industrial system. Professional Edsa digital simulation for the case study has the purpose to determine and to control/correct the operational state of the power system. The steps of the analysis are: AC Object Oriented Load Flow, AC Advanced Power Flow with integrated Motor Torque, AC Load Flow Error Checker, AC Motor Starting Load Flow Method, Standard Motor Torque and Performance.
\end{abstract}

\section{Introduction}

Ensuring the proper operation of the electricity receivers requires compliance with the quality parameters of the electricity, respectively maintaining the voltage and frequency around the nominal value [1-2]. The voltage drop is the algebraic difference of the actual values of the voltages between two points of a network, having the same nominal voltage.

The norms establish the voltage variation limits for a network location (the algebraic difference between the service voltage at that location and the nominal network voltage). Depending on the network type, the voltage deviation has to belong to the voltage variation limits $5 \%-10 \%[3-4]$.

Due to the symmetry of the line, it is possible to study the operation of a single phase, with the help of the single-phase scheme in which the return conductor is a fictitious one and has no impedance [5-6]. Voltage drops exist only on the phase conductor. Different types of network lines exist in practice: three-phase single-consumer line, three-phase line with more concentrated consumers, three-phase line fed from two ends (simple loop), complex looped networks [7-8].

The sizing of electrical networks takes into account restrictions on: maximal voltage drop, heating of the conductors. Depending on the type of network, one of the two conditions is dominant, but it is necessary to be satisfied the technical-economic condition for the power line sizing. The calculation of complex loop networks requires the determination of current and power distribution over different branches of the network. Because they consist of many nodes and have many sources, it is much more difficult to

\footnotetext{
* Corresponding author: mariana.dumitrescu@ugal.ro
} 
determine the power and currents flow. The calculation of complex loop networks is structured on two problems:

- the currents and powers flow is required, when the network parameters are known;

- the sections on different branches sizes are required, when the functioning characteristics of the consumers are known.

The transfiguration method reduces a complex network with several supply buses to a line fed at both ends, through a series of successive transformations. The methods used for the power flow analysis are presented below [7-8].

1. Reduction of a conductor of a certain length and section to an equivalent conductor of another length and section, provided that the load flow and voltage drop do not change.

2. The composition of several branches with different supply voltages, which flow in a node, to a single equivalent line. Considering two branches of a network that have a common bus and different voltages, these can be replaced by a single equivalent, having the equivalent admittance and the equivalent voltage.

3. Throwing loads at nodes. To compose branches in parallel in an electrical network, the loads must be located only at their ends, in the nodes. It is necessary to throw the loads at the nodes, provided that the voltage drop is the same for initial and equivalent scheme.

4. Transfiguration star - triangle.

5 Global methods for the electrical calculation of loop power grids. Given the very large number of unknowns to be determined, mathematical methods and models have been introduced to allow the expression of computational relationships and to achieve automation of calculations. These methods, matrix or topological, describe the configuration of the network through a graph [9-10].

Section 2 shows the schematic of the analysed system. Section 3 presents the simulation, analysis and the object oriented power flow. Section 4 presents the simulation, analysis and the advanced power flow.

\section{Power supply of a delivery system with important consumers}

The analyzed power system (Figure 1) is connected to the national grid system through the $\mathrm{SC} 1$ connection stations with $110 \mathrm{kV}$ voltage. The industrial company has important consumers (over 50\%), with a total power between 5 MVA and 40 MVA. The main consumers are supplied with an average voltage of $6 \mathrm{kV}$.

There are two independent supply sources $100 \%$ redundant, with two different connection points (the two sections of SC1 station) and automation connection of the reserve (AAR) from one supply to the other, in case of one supply failure. The short-circuit power of the national grid system (NGS) is $\mathrm{S}=4000 \mathrm{MVA}$ at each connection point. The power delivery station, which is injecting the electric power from NGS, is located in the center of gravity of the industrial consumers and lowers the connection voltage to the distribution consumers voltage network $6 \mathrm{kV}$. It is a deep-connection type by the connection high voltage line $110 \mathrm{kV}$ (SRA1 - 110/6 kV).

The consumers of $6 \mathrm{kV}$ network are concentrated in the following delivery stations:

- The main delivery station SRA1

- The secondary delivery station PD1

- The secondary delivery station PD2

The main industrial consumers are supplied directly from SRA1, which is the main medium voltage electrical distribution station. The secondary consumers are supplied from two electrical distribution stations (distribution points) PD1 and PD2, these being located in the center of gravity of the respective consumers. 


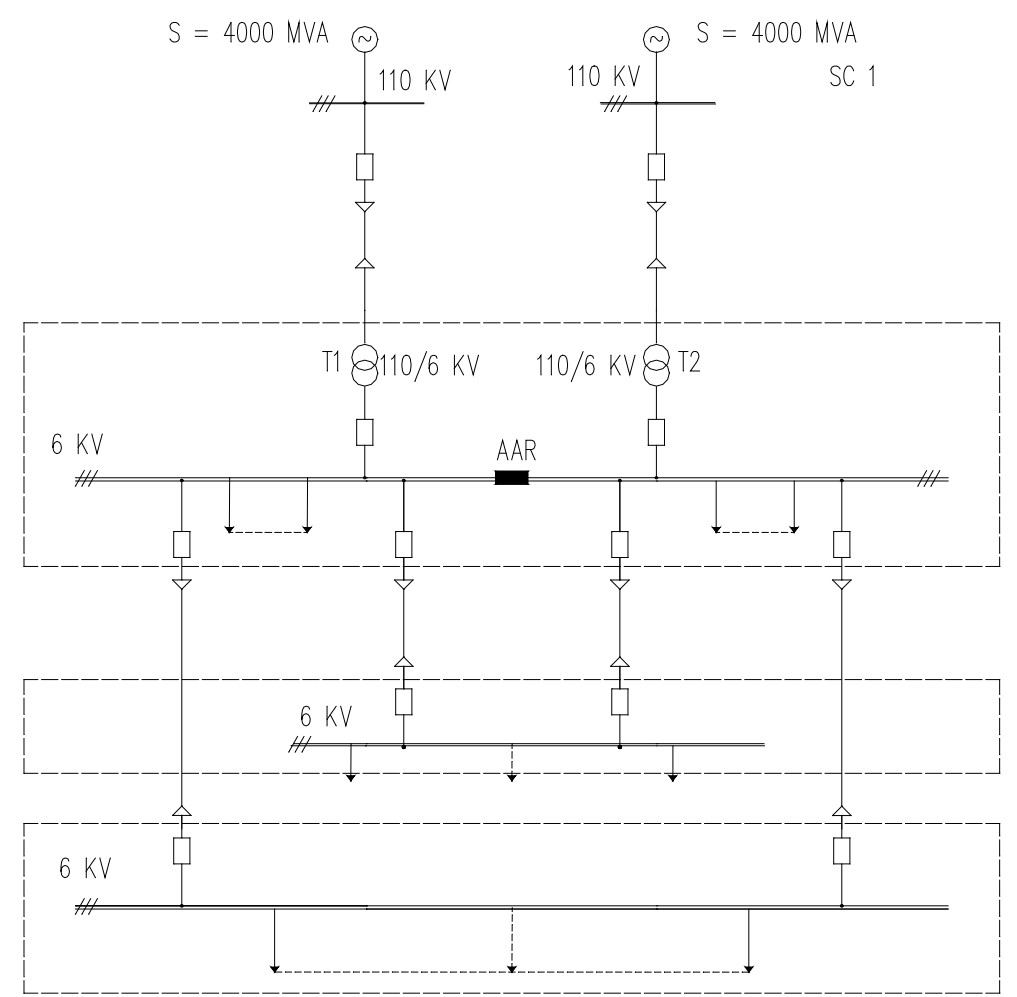

Fig. 1. Power supply of the analysed delivery system with important consumers.

\section{Conducting the power flow analysis}

The important consumers power flow analysis needs professional power systems computer design software to be used, starting with the design phase, but also during the exploitation and maintenance phase [9, 11-12]. Edsa professional software represent a collection of fifty modules/software programs special used for design, analysis, simulation of the electric grid operation and power systems state. Edsa set of tools are used for modeling and computing the network state, they allow solving of the electrical safety, efificiency, quality reliability problems. It covers an important area in the most required industrial application for big power consummers like: electric power transport, electric power delivery, electric power plants, electric power industrial systems. The electric network schematic is easy to draw, which allow to conduct complex simulations and monitoring of the analyzsed power systems.

After the design phase and the implementation in practice of the proposed MV electrical delivery station with important consummers, Edsa profesional modules gives methods and computing algorithms to determine the buses drop voltage, to optimize and maintain the level of voltage, methods to compute the branches power flow, the branches current flow, the transformer loading, the short circuit current analysis (tri phase and mono phase), the torque and performance of the motors. It can help us to determine the most efficient solution of the power flow for the analysed system.

In Figure 1. The MV buses are supplied with the help of two transformers T1 and T2 40MVA. The voltage goes down to $6 \mathrm{kV}$ and supplies the electricity to a complex radial 
electric industrial system. From each of these buses SRA 1 are supplied two secondary stations, buses PD1, buses PD2. From the buses PD1 are supplied 3 air motor-compressors $1,2,3$ and 2 transformers 3, 4 which step down the voltage to $0,4 \mathrm{kV}$ ( they supply $400 \mathrm{~V}$ busbars for 2 asynchronous motors), Figure 2a.

The buses PD2 supply 5 synchronous motors M4, M5, M6, M7, M8 and 2 transformers T5, T6 which step down the voltage to $0,4 \mathrm{kV}$ for other 2 asynchronous motors, Figure 2. The $6 \mathrm{kV}$ delivery power system equipment data are : $\mathrm{T}_{3}, \mathrm{~T}_{4}, \mathrm{~T}_{5}$ and $\mathrm{T}_{6}$ transformers $6 \mathrm{kV} /$ to $0,4 \mathrm{kV}$; busbar 1, busbar 2, busbar 3, busbar 4, busbar 5, busbar 6 and busbar 7, $6 \mathrm{kV}$; syncronous motor M1-8MW; syncronous motor M2-2MW; syncronous motor M35MW; syncronous motor M4, M5, M6, M7 and M8-1,75MW. The 0,4kV delivery power system equipment data are : busbar 8 , busbar 9, busbar 10, busbar 11; asyncronous motor M9-400kW; asyncronous motor M10-400kW; asyncronous motor M11-200kW; asyncronous motor M12-200kW.

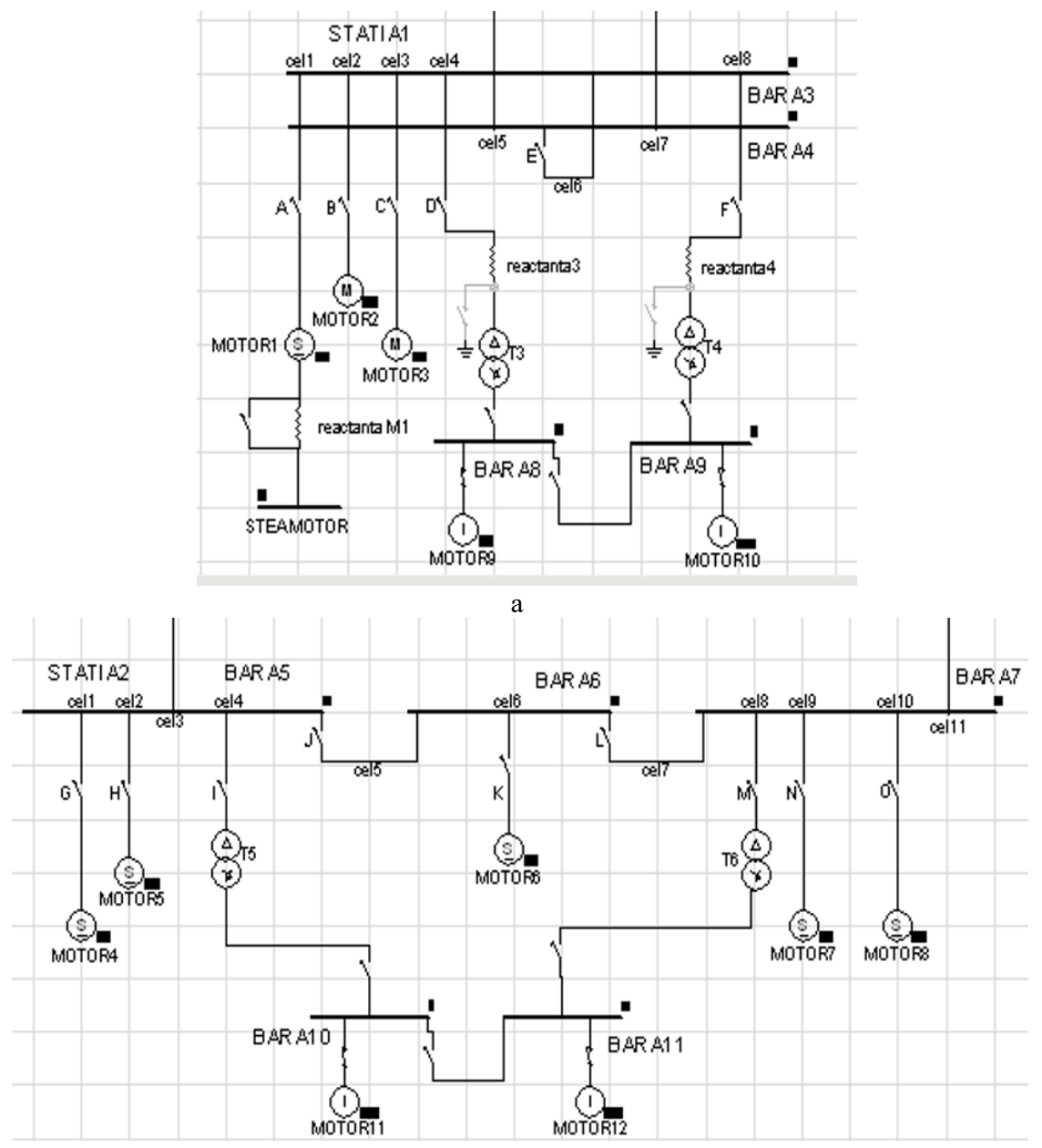

b

Fig. 2. Power delivery radial $6 \mathrm{kV}$ complex system with important consumers, a- important consumers PD1, b- important consumers PD2. 
The menu is giving the results of the proposed analysis the node voltage (Bus Voltage), the branch current (Branch Current Flow), the power circulation on the branches (Branch Power Flow), the transformer load (Transformer Loading), the voltage violation (Bus Violation) ), Current Violation, Transformer Violation. In Figure 3 are presented the current flows and the voltage drops percent $\%$ for $6 \mathrm{kV}$ network and $0,4 \mathrm{kV}$.

\begin{tabular}{|r|l|l|l|}
\hline \multicolumn{5}{|c|}{$6 \mathrm{kV}$} \\
\hline Nr. & From & \multicolumn{1}{|c|}{ To } & Curent(A) \\
\hline 1 & Utility1 & BARA1 & 38 \\
\hline 2 & Utility2 & BARA2 & 39 \\
\hline 3 & BARA1 & BARA5 & 423 \\
\hline 4 & BARA1 & BARA3 & 269 \\
\hline 5 & BARA2 & BARA4 & 1174 \\
\hline 6 & BARA2 & BARA7 & 642 \\
\hline 7 & BARA3 & MOTOR2 & 269 \\
\hline 9 & BARA4 & MOTOR1 & 1101 \\
\hline 10 & BARA4 & MOTOR3 & 74 \\
\hline 12 & BARA5 & MOTOR4 & 212 \\
\hline 13 & BARA5 & MOTOR5 & 212 \\
\hline 15 & BARA6 & MOTOR6 & 214 \\
\hline 16 & BARA6 & BARA7 & 214 \\
\hline 18 & BARA7 & MOTOR7 & 214 \\
\hline 19 & BARA7 & MOTOR8 & 214 \\
\hline 20 & BARA3 & T3 & 13.2 \\
\hline 21 & BARA4 & T4 & 12.5 \\
\hline 22 & BARA5 & T5 & 4.3 \\
\hline 23 & BARA7 & T6 & 6.6 \\
\hline
\end{tabular}
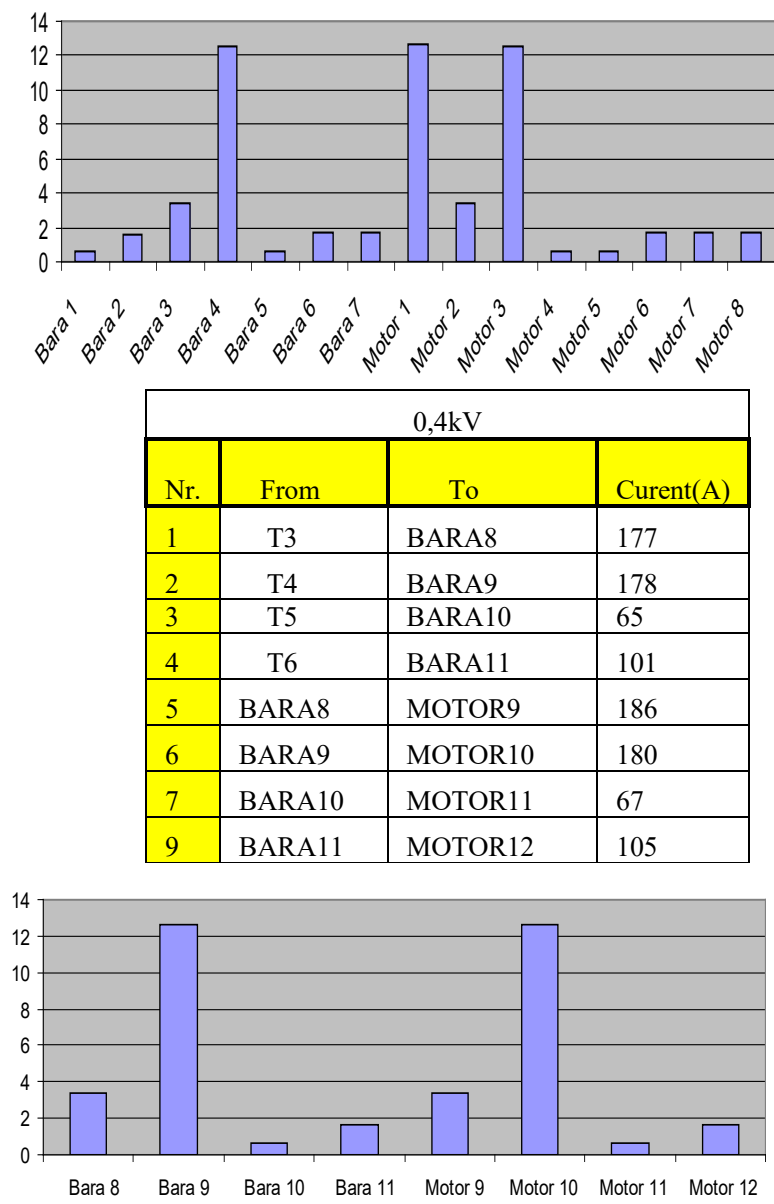

Fig. 3. The branches current flow $[\mathrm{A}]$ and the buses voltage drops $[\%]$ for $6 \mathrm{kV}$ and $0,4 \mathrm{kV}$.

In the figure above we have the current circulation in Amp for each branch as well as the voltage drops on the buses. According with the limits standardized, there are voltage drops violations.

\section{Advanced power flow analysis}

The automatic voltage control can be implemented in the study case analysis by setting the parameters of the transformers. If we want to change the type of socket in the mayor of the T1 transformer, we select the T1 transformer and at the "Load Flow" option we will select the "On" command in the "Automatic Tap Adjustment" menu (Figure 4). We will proceed identically with all the other 5 transformers. As with transformers for automatic voltage 
control, we select each motor and check the "Load Flow" option for "Automatic Voltage control".

After these settings have been made for both transformers and motors, the diagram is running again in the Edsa program and the "Object Oriented Load Flow" command is activated. After running in the "Results" menu we select "Voltage Control".

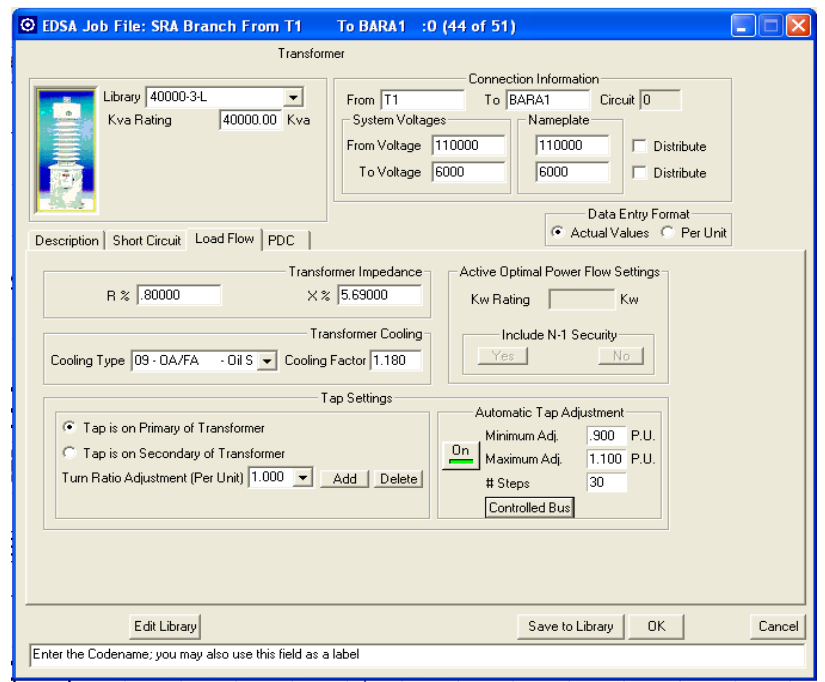

a

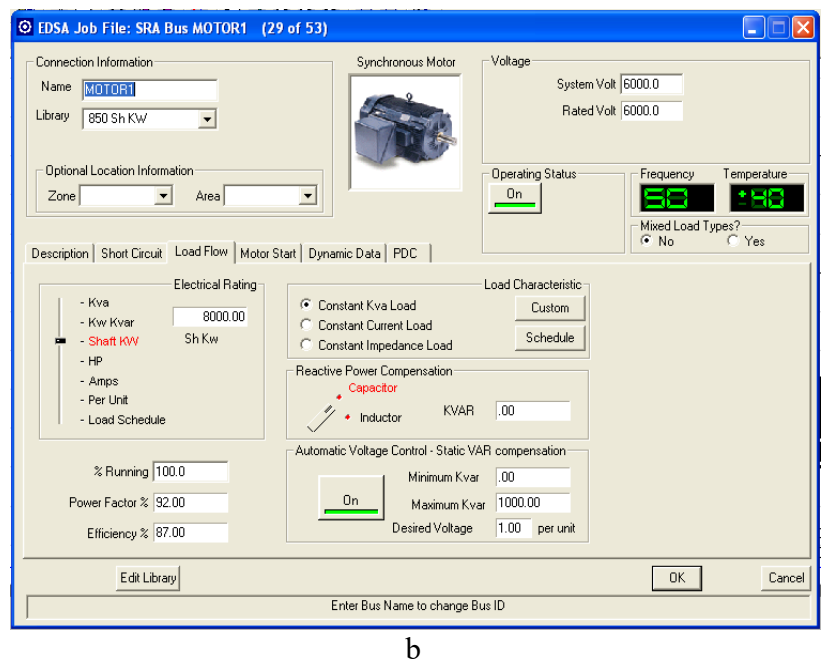

Fig.4. Setting of the automatic voltage control for transformers (a) and motors (b).

The lower voltage limit has been set to 0.95 , so the program is required not to reduce the voltage by more than $5 \%$ of the predefined values. After transferring these values to the diagram, the parameters of each transformer change, the program shows the socket on which the transformer initially worked but also the one on which it must work now. Figure 5 shows us the initial voltage [V] but also the new voltage values [V] improved after setting the automatic control on the both networks buses to $6 \mathrm{kV}$ and respective $0,4 \mathrm{kV}$. 

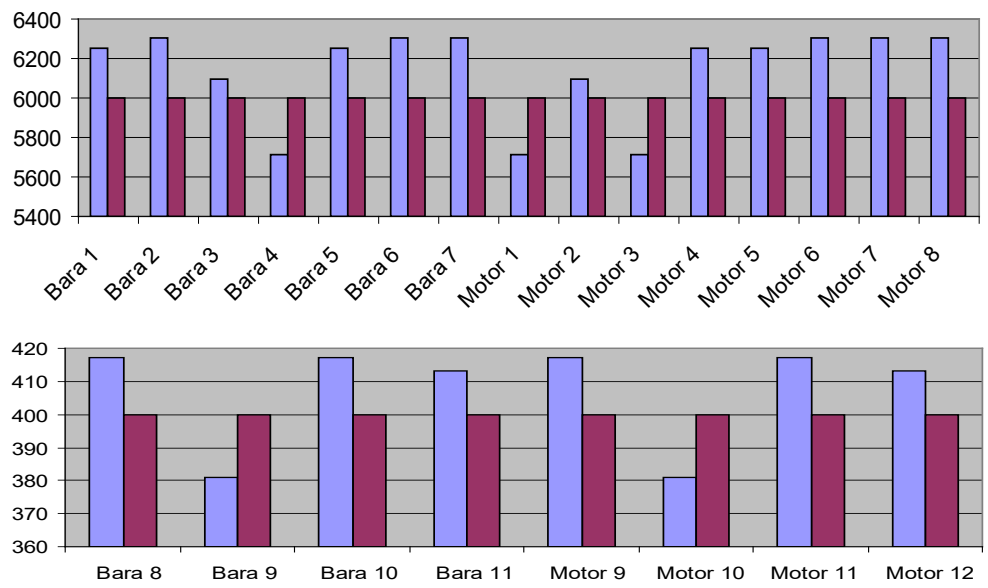

Fig. 5. Voltage $[\mathrm{V}]$ before and after the buses automatic control to $6 \mathrm{kV}$ and $0,4 \mathrm{kV}$.

Beside the standard load flow, we can also simulate the effect of starting the motors in terms of voltage drops. For this the engines are edited: asynchronous motors in the "Motor Start" section, "To be started" option is set to "Full Voltage"; synchronous motors in the "Motor Start" section, "To be started" option is set to "Resist/Reactance \%", Figure 6.

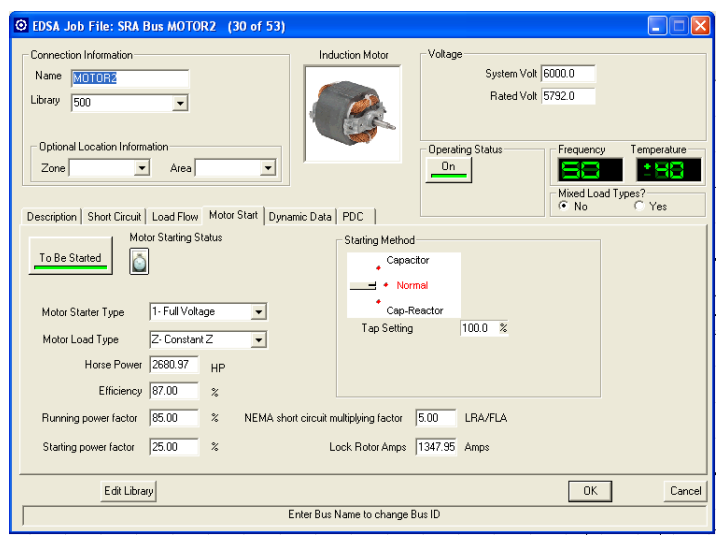

a

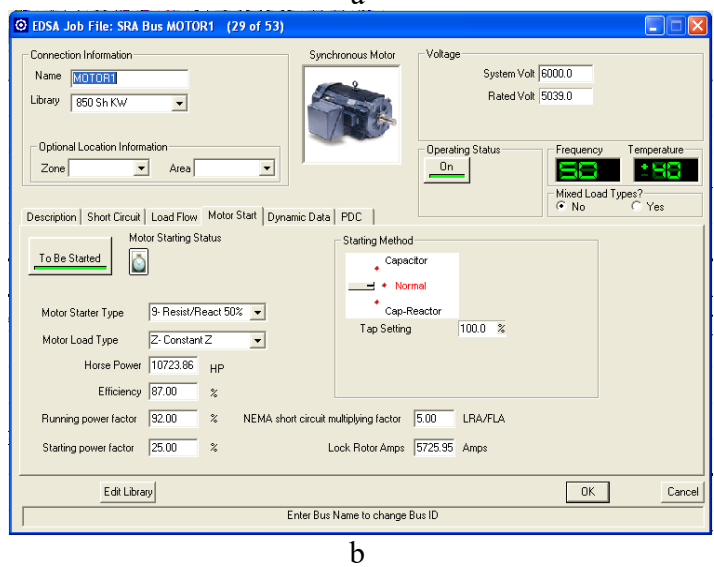

Fig. 6. Setting Motor Start power flow options asynchronous (a), synchronous motors (b). 
From the Edsa toolbar we can select the "Motor Starting" option. At this point the Edsa program checks the convergence of the calculation iterations before, during and after starting the engine. From the "Results" menu, we select "Motor Starting" and the table below shows the motor voltages before starting, during starting and after starting.

Table 1. Motor Starting module results-voltages before starting/during starting/after starting.

\begin{tabular}{|c|c|c|c|c|c|c|}
\hline \multirow[b]{2}{*}{ ID } & \multirow[b]{2}{*}{ Node Label } & \multirow{2}{*}{$\begin{array}{l}\text { Power } \\
{[\mathrm{KW}]}\end{array}$} & \multirow[b]{2}{*}{ Start Method } & \multicolumn{2}{|c|}{ Voltage $[\mathrm{V}]$} & \multirow[b]{2}{*}{ After } \\
\hline & & & & Before & During & \\
\hline 1 & MOTOR1 & 8000 & Res/Reac 50\% & 6400.0 & 4038.9 & 5771.0 \\
\hline 2 & MOTOR2 & 2000 & Full Voltage & 6274.5 & 5024.3 & 6076.5 \\
\hline 3 & MOTOR3 & 500 & Full Voltage & 6400.0 & 4043.1 & 5772.4 \\
\hline 4 & MOTOR4 & 1750 & Res/Reac $80 \%$ & 6274.5 & 6015.3 & 6236.7 \\
\hline 5 & MOTOR5 & 1750 & Res/Reac $80 \%$ & 6274.5 & 6015.3 & 6236.7 \\
\hline 6 & MOTOR6 & 1750 & Res/Reac $80 \%$ & 6400.0 & 5967.6 & 6311.3 \\
\hline 7 & MOTOR7 & 1750 & Res/Reac $80 \%$ & 6400.0 & 5967.7 & 6311.3 \\
\hline 8 & MOTOR8 & 1750 & Res/Reac $80 \%$ & 6400.0 & 5967.7 & 6311.3 \\
\hline
\end{tabular}

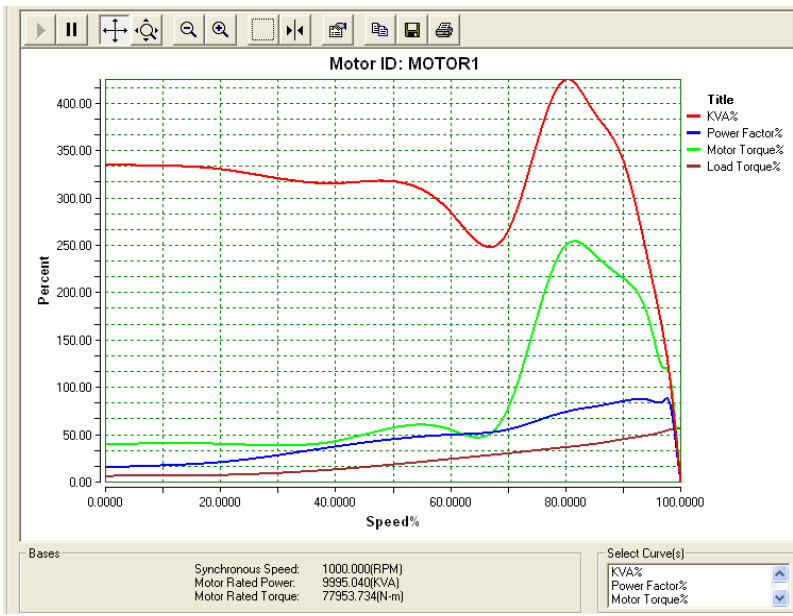

a



$\mathrm{b}$

Fig. 7. Advanced starting dynamic performance motor parameters motor 1 (a), motor 2 (b). 
For advanced starting and motor performance we choose an operation case, considering that only Motor 1/Motor 2 works and the other engines are ready to start. The Motor is edited, switching from the "To be started" to the "Running" mode, the other engines remaining on the "To be started" mode. We select the "Motor dynamic information" and the Calculation option to compute the motor parameters (Figure 7).

\section{Conclusions}

With the help of a professional software like Edsa it is possible to analyze, from the point of view of safety in operation any type of network, whether three-phase or single-phase. This program is working according to IEC and IEEE standards. It helps to easily build the model of a complex networks, like the real study case of an industrial power delivery system with important consumers, we presented in this paper.

With a possibility of unlimited number of nodes and 45000 symbols, with an important data base for the equipment, the project can be organized on several pages or drawings. After choosing and implementing a medium voltage network in the Edsa program, it has a variety of modules with calculation methods and algorithms for determining voltage drops of the buses as well as methods for correcting them, improving the power flow until an optimal solution. Load flow analysis gives detailed information about branches currents flow and violations, the buses voltages and violations, the loading of equipment like transformers and violations, the control of the power flow, the automation control for improving of the voltage drops, the torque and engine performance, but it helps also for determining of the most economical load circulation solution.

\section{References}

1. *** IEC 61000-3-7, Part 3: Limits - Section 7: Assessment of emission limits for fluctuating power in MV and HV power systems (2000)

2. *** IEEE P1366, Trial Use Guide for Electric Power Distribution Reliability Indices, Working Group on System Design, Draft 5, Ian. (2001)

3. $* * *$ IEC 61400-21, Measurement and assessment of power quality characteristics of grids (2005)

4. *** IEEE Standard 519-1992 Power Grids Distortion Limits. Voltage characteristics of electricity supplied by public distribution systems, European Norm EN 50160, Nov. (1999)

5. R. C. Dugan, H. W. Beaty, M. F, McGranagham, Electrical power systems quality (New York: Mc.Graw-Hill, 1996)

6. M. Bazrafshan, N. Gatsis, Comprehensive modelling of three phase distribution systems via the bus admittance matrix. IEEE Trans. Power Syst. 33, 2015-2029, (2018).

7. M. Dumitrescu, Power Systems (Edited on Didactica and Pedagogica, Bucuresti, 2002)

8. M. Dumitrescu., Efficiency and Reliability on Power Systems (Edited on Didactica and Pedagogica, Bucuresti, 2003)

9. N. Mohan, T. M. Undelan, W.P. Robbins, Power systems, applications and design (2nd Edition New York, Wiley \& Sons, 2005)

10. M. Saied, Measures for the supply efficiency in distribution network, INT.J.Elect. Power Energy Systems, vol 23, 427- 434, (2001)

11. A. Garces, A linear three phase load flow for power distribution systems. IEEE Trans. Power Syst. 31, 827-828, (2016)

12. B. Sereeter, F. Vuik, C. Witteveen, Newton Power Flow Methods for Unbalanced Three-Phase Distribution Networks. Energies 10, 1658, (2017) 